This item was submitted to Loughborough's Research Repository by the author.

Items in Figshare are protected by copyright, with all rights reserved, unless otherwise indicated.

\title{
Trajectory generation for autonomous soaring UAS
}

\section{PLEASE CITE THE PUBLISHED VERSION}

http://link.springer.com/article/10.1007/s11633-012-0641-5

\section{PUBLISHER}

(C) Springer Verlag and Institute of Automation, Chinese Academy of Sciences

\section{VERSION}

AM (Accepted Manuscript)

\section{LICENCE}

CC BY-NC-ND 4.0

\section{REPOSITORY RECORD}

Clarke, Jonathan H.A., and Wen-Hua Chen. 2012. "Trajectory Generation for Autonomous Soaring UAS". figshare. https://hdl.handle.net/2134/11139. 
This item was submitted to Loughborough's Institutional Repository (https://dspace.lboro.ac.uk/) by the author and is made available under the following Creative Commons Licence conditions.

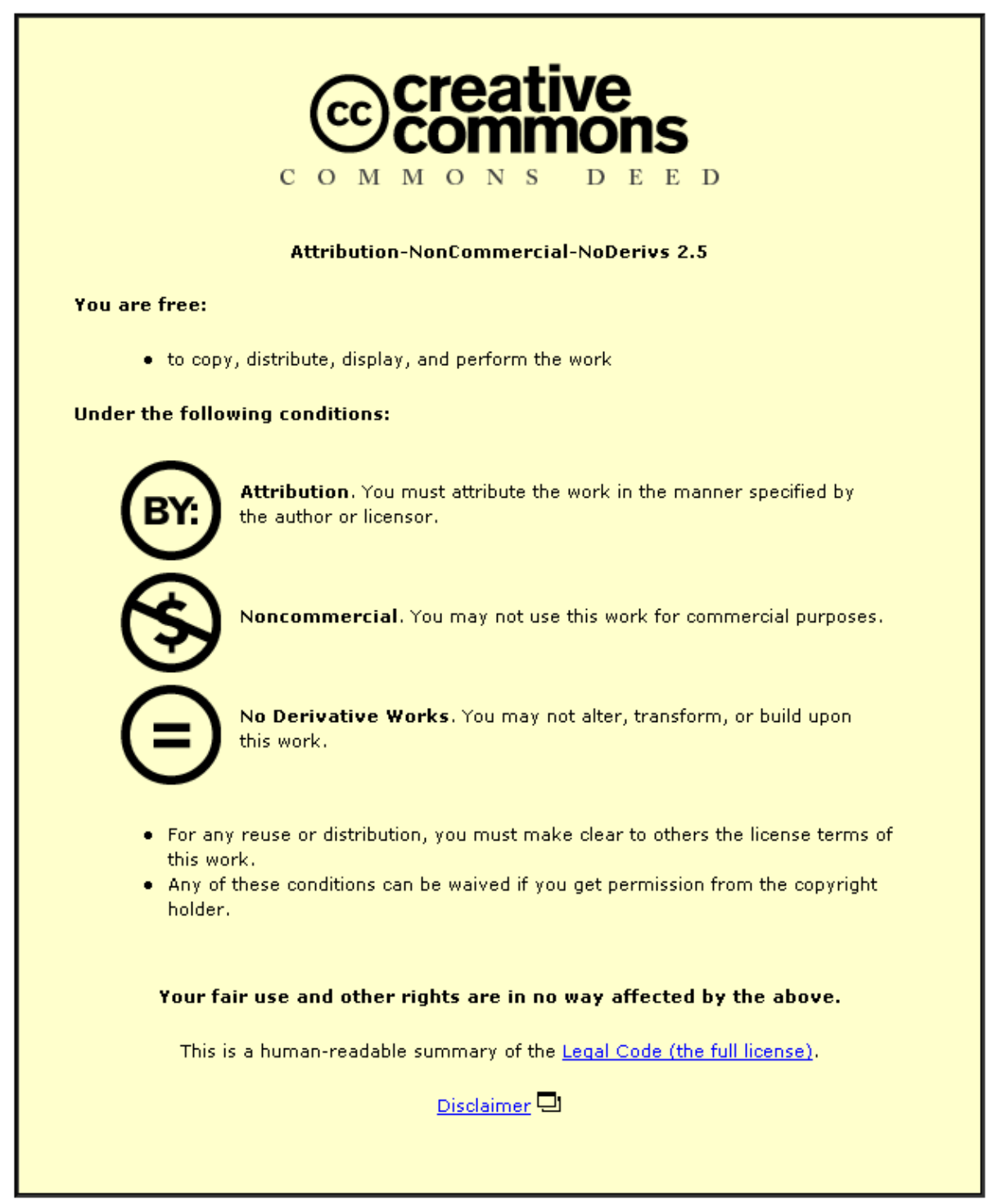

For the full text of this licence, please go to: http://creativecommons.org/licenses/by-nc-nd/2.5/ 


\title{
Trajectory Generation for Autonomous Soaring UAS
}

\author{
J. H. A. Clarke and W-H. Chen \\ Department of Aeronautical and Automotive Engineering, \\ Loughborough University \\ Loughborough, UK \\ J.H.A.Clarke@lboro.ac.uk
}

\begin{abstract}
As unmanned aerial vehicles are expected to do more and more advanced tasks, improved range and persistence is required. This paper presents a method of using shallow layer cumulus convection to extend the range and duration of small UAVs. A simulation model of an XModels XCalubur electric motor-glider is used in combination with a refined $4 \mathrm{D}$ parametric thermal model to simulate soaring flight. The parametric thermal model builds on previous successful models with refinements to more accurately describe the weather in northern Europe. The implementation of the variation of the MacCready setting is discussed. Methods for generating efficient trajectories are evaluated and recommendations are made regarding implementation.
\end{abstract}

UAV, UAS, Soaring, Tragectory, thermal, Optimal, Heuristic

\section{INTRODUCTION}

Over the last two decades the use of unmanned aerial vehicles (UAV) has exploded. As the use of UAVs has increased the demands placed upon the platforms have also increased. Simultaneously people desire greater access to flying assets lower down the chains of command; whether that is for military purposes or civilian survey work. This requirement necessitates the use of smaller aircraft without loss of performance. Typically the limiting factor for these small UAVs is short flight duration, limited range and payload. Many activities such as forest fire monitoring, border patrol, atmospheric research, communication relays and other surveillance tasks require greater persistence from the airframe used. Although advancements in engine and battery technology, along with miniaturisation of much of the on-board systems, continue to provide performance and capability improvements there is still a need for the introduction of novel methods to improve the range and persistence of the aircraft. One such novel solution is the extraction of energy from naturally occurring phenomena such as atmospheric convection.

Techniques to extract energy from shallow layer cumulus convection have been employed by full-size glider pilots to increase their range and duration for nearly 100 years. These soaring techniques have historically been ignored by the surveillance community because the differences in aircraft wing loading, operating speeds and efficiency rendered them pointless.
However with the latest generation of UAV this is no longer the case.

Although soaring techniques have been investigated from before the 1930s there application to UAV is a relatively new field. Wharington [1] was the first to propose that autonomous soaring could be a viable method for extending UAV performance (range, endurance and usable payload capacity) in 1998. Since Wharington first proposed that static soaring was a viable option guidance algorithms have been developed using reinforcement learning and a neural-based thermal locator to detect and utilize thermals [1]. The results showed that both heuristic controllers [2] [3] and reinforcement learning could be effectively combined with a thermal locating algorithm to improve UAV performance. Algorithms utilising reinforcement learning have proved too computationally expensive for real-world application, leaving robust but heuristic algorithms the only option with current processing power. These simple heuristic algorithms have been successfully employed [2][3] but there still is a desire to further optimise the aircraft trajectories.

For progress to be made in the improvement of the methods used to extract energy from atmospheric convection it is advantageous to start in a simulation environment before moving on to real-world flight tests. The use of a simulator allows the algorithms to be tested in a controlled environment where the conditions are both fully understood and repeatable. However for the results of the simulation to be both be meaningful and useful the simulation environment must be realistic. Three key areas of the simulation environment need to accurately reflect reality; the aircraft flight dynamics, the atmospheric model and the aircraft flight control structure.

The following three sections deal with the aircraft flight dynamics, the atmospheric model and the aircraft flight control structure respectively.

Having established the simulation environment, section five and six shows how suitable atmospheric convection can be identified and exploited. Section seven gives some pertinent results. Section eight highlights the key conclusions and recommendations for real-world implementation. 


\section{AIRCRAFT DYNAMICS MODEL}

Autonomous soaring can be simulated with rudimentary knowledge of the aircraft in question but in order to optimise the algorithms an accurate model of the aircraft in question is required. The type of aircraft is unimportant for the purpose of optimisation as long as the actual aircraft is reflected. The X-Models XCalibur was chosen as the test aircraft as it has the best performance of all the aircraft available at Loughborough University. The X-Models XCalibur is a self launch electric glider with a $3.2 \mathrm{~m}$ span and a typical take-off weight of $3.5 \mathrm{~kg}$, giving a wing loading of approximately $120 \mathrm{~Pa}$. The XCalibur was developed from X-Models F3J competition aircraft, and as such has a performance comparable with larger gliders used by other researchers [2][3]. An accurate model of the aircraft in use also facilitates the stabilisation and control algorithms to be validated in conjunction with the high-level trajectory generation algorithms.

It was decided to have a non-linear model that included the stall behaviour of the aircraft. This is important because of the possibility that the combination of the speed to fly and path planning algorithms might cause the aircraft to fly close to the stall condition. If the aircraft did stall, it is important to know how the control structure would behave.

The chosen environment for constructing the dynamics model was Matlab / Simulink. The dynamics model is made up of four distinct parts; left and right wings, elevator and rudder. The fuselage is neglected in the calculations because of its small influence in relation to the aircraft's responses and the difficulty involved in modelling it accurately. The glider dynamics model also includes a model of the propeller-motor combination, providing data for the simulation of the initial launch procedure and any subsequent powered flight that may be required. The model includes a power drain model allowing the battery consumption to be approximated.

The coefficients used in the model presented are based on the performance parameters of the XCalibur used at Loughborough University, as confirmed by flight tests.

\section{SHALLOW LAYER CUMULUS CONVECTION}

In order to simulate and ultimately attempt to optimise the aircrafts trajectory, it is necessary to have a model of the atmospheric structures that the aircraft is flying through. Although ideally a model of high fidelity should be used to simulate and optimise the control algorithms, in reality a relatively crude model can be used effectively, provided that it can reflect the salient characteristics of the updraught structures.

Once the vertical motion of shallow layer convection is sufficiently strong to support the continued flight of an aircraft it is referred to as 'a thermal'. Nearly all of the existing models of thermals have been produced for hotter countries than the UK, as a result it is necessary to modify parameters to more fully reflect the differing conditions.

\section{A. Thermal Profiles}

The 'British Standard Thermal' (BST)[4] that is used by the British Gliding Association (BGA) is given in the international system of units (SI) in (1).

$$
\mathrm{w}=2.16\left[1-(\mathrm{r} / 304.8)^{2}\right]
$$

The magnitudes used in the British standard thermal (BST) will be used as a starting point to base the models on. It is worth stating that the BST is not a mean thermal for Britain but is an optimistic case that the BGA uses to assess full-size glider performance. The mean thermal strength that Allen [5] detected at Desert Rock in July of 2002 was $2.69 \mathrm{~m} / \mathrm{s}$ with a maximum strength of $6.3 \mathrm{~m} / \mathrm{s}$. It is therefore suggested that the BST may represent a typical thermal on a summer's day in Britain.

Although the BST is a good starting point, it does not model atmospheric or localised sink, or provide any information on the variation of strength or radius of the thermal with height. Nor does the BST provide any information about the time dependent nature of the thermal. Once sufficiently centred in a thermal this detail has a negligible effect. However, when considering methods of centring it is advantageous to consider the foregoing factors.

It has long been known that downdraughts are associated with shallow layer cumulus convection [6] [7]. Allen chose to largely ignore the associated downdraughts they were included when he arrived at a family of parametric profiles, as shown in Fig. 1 [5]. Another parametric profile that has been used is that of Wharington [1], shown in Fig. 2. Although this model is less accurate than others [2] [8], it lends itself well to mathematical analysis [3].

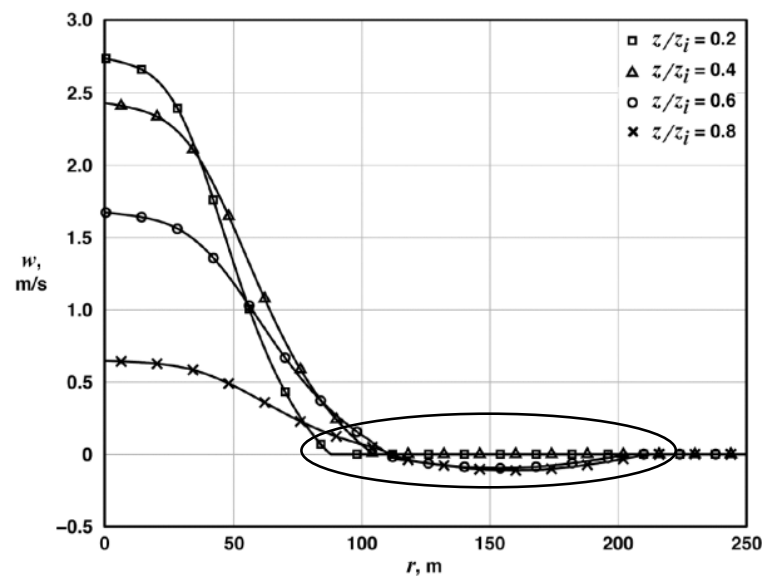

Figure 1. M.J. Allen - Vertical Thermal Velocity Prediction at height ratio of 0.4 [5] 


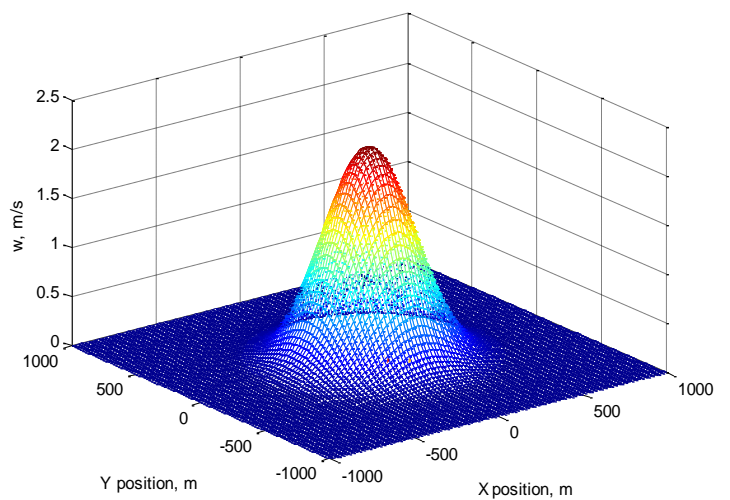

Figure 2. Thermal Lift Distribution as used by Wharington [1] [3]

None of the existing thermal distributions found meet what is felt to be a suitable starting distribution. The distributions either have spurious maxima or have unrealistic sink associated with them. Allen's model was the best found but that did not, for the most part, include sink. Allen [5] used atmospheric data to generate the core velocities but his prediction of sink does not match the evidence of the almost inevitable presence of localised sink around the thermals found in the UK, as described in [7], [9] and [10]. The sink shown in Fig. 3 is exaggerated for illustrative purposes.

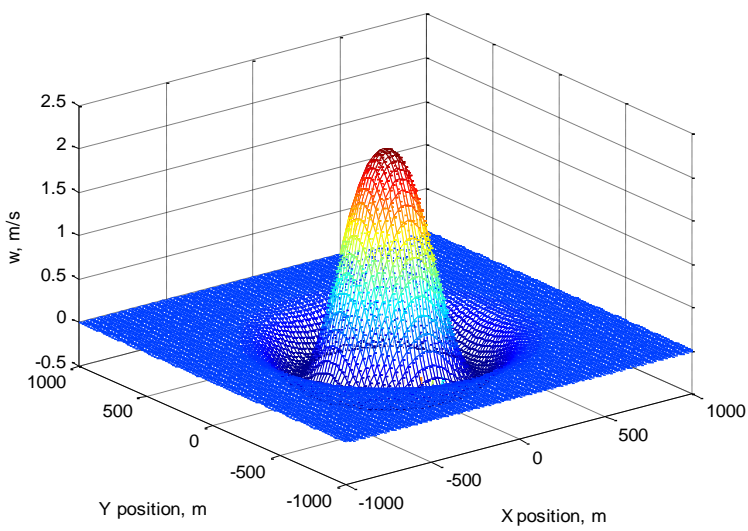

Figure 3. Proposed British Thermal Lift Distribution

Most models do not have sink associated with them [1] but instead rely on conservation of mass to determine atmospheric sink. Although there is no denying that conservation of mass does apply to the global atmosphere, it may be argued that local weather systems will have a larger effect [9]. It can be frequently observed that regions of high pressure, anticyclones, effectively suppress thermal formation over large areas of the country. Additionally, mountain wave and Cloud street formation both have a strong influence on thermal formation. It is also known that strong down drafts form separately to thermals. Conservation of mass is a good starting point for the atmospheric map but for large maps, overlying a large period sinusoidal distribution for atmospheric wave or 'cloud street' formation yields improved realism [7] [10] [6].

As all the models looked at fall short in one way or another, a new thermal velocity distribution model is proposed as shown in Fig. 3. The model derived follows the profile as measured by Allen [5] with the addition of the localised sink and is given in (2) bellow.

$$
w=w_{\max }\left(-C_{1}\left(\frac{r}{\sqrt{C_{1}} r_{\text {inner }}}\right)^{2}+1\right) e^{\left(-\left(\frac{r}{\sqrt{C_{1}} r_{\text {inner }}}\right)^{c_{2}}\right)}
$$

$\mathrm{C}_{1}$ and $\mathrm{C}_{2}$ control the radius and magnitude of the sink associated with the thermal structure. $w$ and $w_{\max }$ are the current and maximum vertical velocity of the core of the thermal. $r$ is the current radial location of the aircraft, with $r_{\text {inner }}$ being the core, updraft, radius.

Shallow layer cumulus convection is a ring vortex structure and a result also has equivalent horizontal movement of air associated with it. This movement, although considerable, is small when compared with speed of the aircraft. The horizontal movement of the air is not taken in to account in this model.

\section{B. Thermal Spacing}

Lenschow [11] derived an equation capable of estimating the distances between thermals at a constant height ratio, $\frac{z}{z_{i}}$, of 0.4 . Where, $z_{i}$ is the height of the convective layer and $z$ is height. A guide to the distances between the thermals was given as 1.5 to 2.5 times the convective scale by Wallington in Meteorology for Glider Pilots [9]. John Delafield [6] suggested that the distances were between 2 and 3 times the convective scale in his book 'Gliding Competitively'. This would tend to suggest that the thermal spacing to height ratio is not consistent between different climates. An explanation of this phenomenon may be that there is a minimum spacing for the formation of thermals; otherwise they would merge. In warmer climates the characteristic convective length scale will be much more than that of a temperate climate. The reduction in spacing of thermals in a temperate climate, although less than in warmer climates, is not sufficient to maintain an equivalent thermal spacing to convective length scale ratio. Following the anecdotal evidence from [9] and [6], Lenschows' equations can be reworked using the numbers proposed above to take the following forms for a British climate.

$$
\frac{N z_{i}}{L}=0.5
$$

$\mathrm{N}$ is the number of updrafts encountered over a length $\mathrm{L}$. This equation can be rearranged to give the number of updrafts in a given area.

$$
N=\frac{0.25 X Y}{z_{i} r_{\text {outer }}}
$$


Where, $r_{\text {outer }}$ is the outer diameter of the average thermal. $\mathrm{X}$ and $\mathrm{Y}$ are the length and breadth of the area of interest. Although there is no way of verifying (3) and (4) without more accurate flight data, the predictions are more typical of the British weather pattern.

Lenschow [11] also derived equations that predict the variation of the thermal structures strength and radius with height. These equations are employed without alteration. The thermal is assumed to be a bubble rising at the dry adiabatic rate.

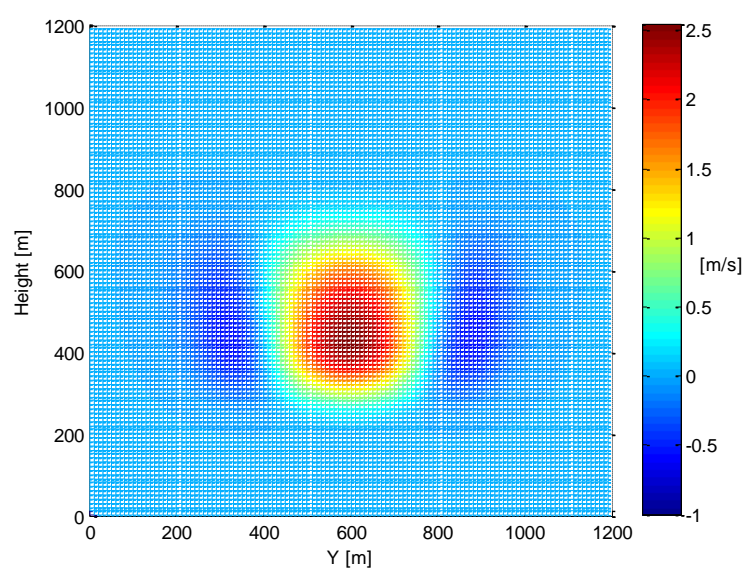

Figure 4. Thermal Cross Section

The positioning of the thermals on a given map is often given as random. On occasions when the thermals have no obvious trigger this is a fair assumption. However, in the British Isles the thermals often do have trigger points; a dark field, a power station, a factory, a motorway etc. The effect of trigger points is to set up streets of thermals up and down wind from the last. This cloud phenomenon is also described as cumulus mediocris radiatus. Knowledge of this phenomenon can be used to aid the autonomous decision making processes. Using Lenschows' equations as modified in above in combination with random noise the thermal trigger map shown bellow in Fig. 5 is generated.

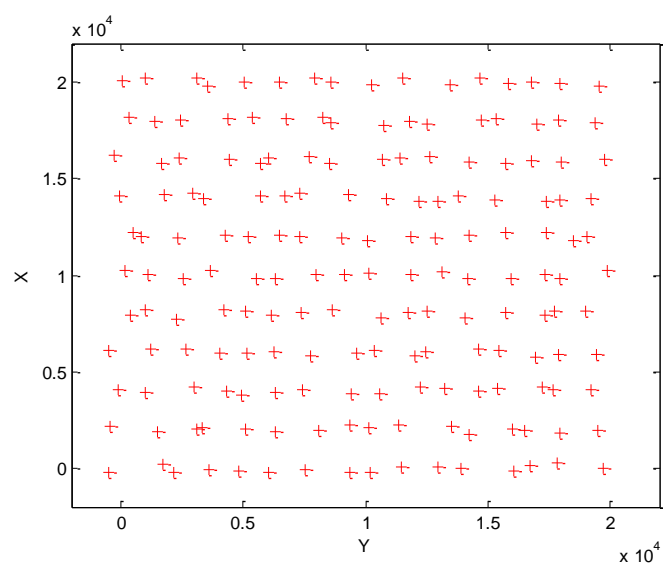

Figure 5. Thermal Triggers
At first glance this is very dense and regular map, but after the trigger times are distributed the map is as shown in Fig. 6. It can be seen that Fig. 6 has an almost random quality despite the map being well structured. This provides insight into why the assumption of randomised thermal positions on a 2D map provides a good likeness. The map used in the simulations presented here uses a 4D map, with the position and strength of the thermals varying with time.

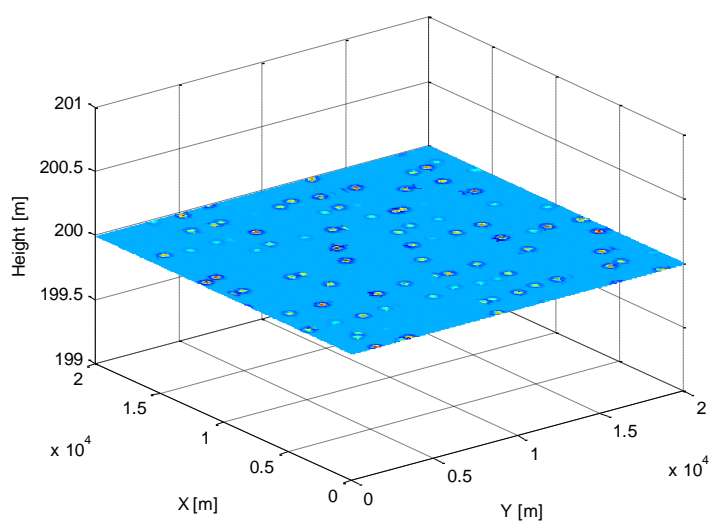

Figure 6. Thermals at $200 \mathrm{~m}$ at $\mathrm{t}=0$

\section{CONTROL STRUCTURE}

The flight control system is critical to the successful execution of any generated trajectory. While the limitations of the flight control system will always impose constraints on the trajectory generation algorithms, it is advantageous to maximise the flight control performance to keep these practical limitations to a minimum.

For the trajectories generated to be useful it is imperative that the flight control system be representative and have comparable performance to the real platform. The flight control system used on the real aircraft is based on a nested PID architecture; the simulation was setup to reflect this. The controller features a novel use of feed forward control as part of the pitch and yaw controllers. This is to pre-emptively suppress fluctuations in pitch and yaw; due to uncoordinated turns and adverse yaw. As these parts of the controller are a simple form of model predictive control they are more platform dependent than the other parameters. 


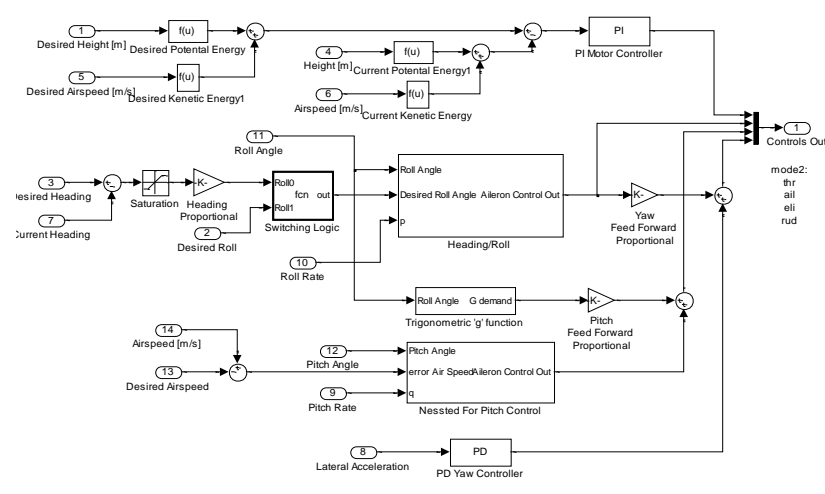

Figure 7. Control Structure

This control structure is not designed to reflect an optimal solution but instead accurately reflect the performance capabilities of the flight control structure on the aircraft in question. In many cases the control system is only tuned for adequate performance.

\section{CHOICE OF AND EVALUATION OF THERMALS}

The identification of suitable thermals has been investigated in detail over the years [3] [7] [9]. Equations to predicting the optimum speed to fly to optimise overall cross country speed are well known [4] [12] but are incomplete without an estimate of the strength of the next thermal to be encountered; which is of course unknown until it is encountered. This prediction is generally referred to as the 'MacCready setting' after the first person to pose this problem. The choice of MacCready setting is a frequent topic of conversation at gliding clubs, but the problem boils down to how much risk can be tolerated. As the setting is a function of risk it follows that the setting is related to height, as a higher aircraft has a greater probability of encountering another thermal with the associated reduction in the risk of a forced landing or the use of powered flight or a powered climb. Edwards [3] viewed landing out as unacceptable and his MacCready function reflects this. Others have a higher tolerance to risk and as such select a more aggressive MacCready function. The XCalibur is fitted with a powerful electric motor so if the mission demanded maximum cross country speed at all cost, the MacCready function could be set aggressively resulting in profile that would ignore all but the strongest thermals, necessitating the use of the motor. This powered climb and glide profile would be extremely power hungry, reducing range and crippling endurance. The choice of the function ultimately depends on the aircraft in question and the mission profile with the associated constraints. A good example of a constraint would be a maximum allowable height during the flight. The effect of this constraint would be to cause the MacCready function to tend to infinity at that height. In reality there are tolerances and safety margins. This type of constraint is shown in Fig. 8.

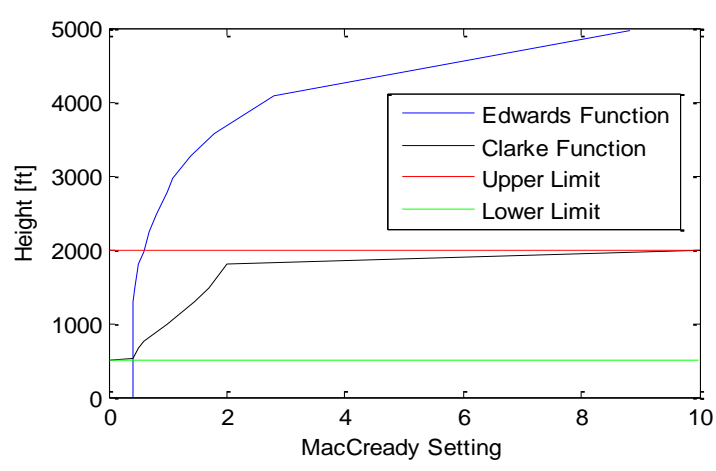

Figure 8. MacCready Setting

Once the MacCready setting has been established the aircraft can fly at the appropriate speed for the conditions and asses any thermals encountered for suitability. To facilitate the correct identification of thermals two variometer readings are used; an instantaneous reading and an averaged reading. The averaged reading suppresses the influence of turbulence and helps to prevent the erroneous thermal detection. Once a thermal stronger than the MacCready setting is detected the soaring algorithms are triggered. At that stage the averaged reading must drop bellow a lower critical value before the search for lift is abandoned. This is necessary because the aircraft will take a few turns to find the core of the thermal, with the sink that exists around the edge the average reading may fall considerably before restabilising. The instantaneous reading is used to position the aircraft in the thermal. If the average reading drops below the MacCready setting, the thermal is left in the hope of finding a stronger thermal along track. Similarly final glide calculations can also affect the MacCready setting although this will not be considered further. A discussion of how to select a MacCready Function is presented in [13].

To maximise the aircraft's chances of finding thermals and thus minimising the likelihood of unnecessary energy expenditure it is possible to utilise the cloud street phenomena. To do this once a thermal has been found and utilised the trajectory is modified to fly directly into or downwind as long as this does not take the aircraft more than $90^{\circ}$ off-track. The soaring controller also includes a prediction of the likely next thermal location along the current cloud street. If the location of this thermal would take it more $90^{\circ}$ off-track the cloud street is also rejected. This projection is based on the convective scale assumptions presented in section III b. The use of cloud streets does however have a penalty as it increases the total distance flown. The worst case is a flight with the wind at 45 degrees to the desired track, this scenario results in a $41 \%$ increase in the total distance flown. This increase in distance can be controlled by lowering the track angle deviation allowed, but again reducing this deviation angle increases the likelihood of needing the motor. 


\section{CENTRING WITHIN A THERMAL}

As time and height invariant thermal models aid visualisation, these have been used to illustrate the soaring techniques. However, accurate atmospheric models are required to validate the proposed techniques.

A thermal can be viewed as a vortex ring travelling upwards through the atmosphere with the aircraft's objective to be carried aloft in said vortex. In order to maximise the potential height gain of a given thermal the aircraft has to centre in the thermal as quickly as possible. If the aircraft does not find equilibrium inside the core of the thermal, then the aircraft will drop out of the bottom of the thermal. The factors that affect the aircraft's ability to find equilibrium include; the up draught strength, size, or inability to locate the strongest lift.

The inclusion of the associated 'sink' around the edge of a thermal is often neglected [1] [2] [3] because the sink found around the edge of very strong thermals is relatively small. However, in colder climates where the rise rate of the thermal may be lower compared to the vortisity of the thermal, the sink around the thermal may be considerable. Once the aircraft is sufficiently well centred in the thermal the presence of sink around the edge of the thermal may be ignored but in order to evaluate the ability of a given algorithm to efficiently centre on a thermal the sink has a profound effect on the success rate.

There are many methods for centring in a thermal but two of the most widely used are the Piggot and the Reichman techniques. Cowling [14] concluded the following:

"The point mass model simulation earlier demonstrates that Piggot's technique works well for negligible lag times and with perfect knowledge of the air mass velocity around the vehicle. For the full simulation model however, it appears that despite using accelerometers the response time is sufficiently long for Reichmann's technique to be more applicable than that of Piggot." [14]

It is therefore logical to base further work on Reichmann's technique. As discussed earlier, the time taken to centre in the core of the thermal is critical to the successful exploitation of the thermal encountered. As a result there is a desire to both better understand and to further optimise the positioning algorithms.

Ensuring the aircraft always turns in one direction while soaring allows the operator on the ground to quickly assess the flight mode the autopilot is currently in. It was also shown by Piggott [7] that reversing the direction of the turn in a thermal is un-advisable. Therefore a turn direction monitor was added so that once a turn direction was chosen, it was not reversed.

Although there are algorithms to detect the relative location of the thermal with respect to the aircraft they are not infallible. This leads to a worst-case scenario of the aircraft turning in the wrong direction once encountering the edge of a thermal. This is the scenario that will be investigated when considering the stability of thermal location algorithms.
The control implemented in the simulation presented is a relatively simple implementation of the Reichman method. Loughborough University operates a range of advanced autopilots. The autopilot that is fitted in the XCalibur is capable of accepting bank angle commands. This facilitates a more straightforward implementation of the Reichmann method than previous possible [2] [3].

Although the Reichman method provides good results, Allen [2] showed improvements by adding a thermal position estimator. The soaring controller therefore takes the following form shown bellow.

$$
\left[\begin{array}{ll}
X_{E s t} & Y_{E s t}
\end{array}\right]=\left[\begin{array}{ll}
\frac{\left(\frac{1}{K s+1}\{\dot{E} \mathrm{X}\}\right)}{\left(\frac{1}{K s+1}\{\dot{E}\}\right)} & \frac{\left(\frac{1}{K s+1}\{\dot{E} \mathrm{X}\}\right)}{\left(\frac{1}{K s+1}\{\dot{E}\}\right)}
\end{array}\right]
$$

$\mathrm{X}$ and $\mathrm{Y}$ are current grid positions and $\dot{E}$ is the rate of change of aircraft energy; Variometer reading. Although (5) is in continuous time form it can be readily discretised for implementation with the real autopilot. This form of prediction has the advantage of having a variable filter length, for the prediction of the location of the thermal. The filter gain, $\mathrm{K}$, was chosen as the time to complete one soaring turn, but this does not have to be the case.
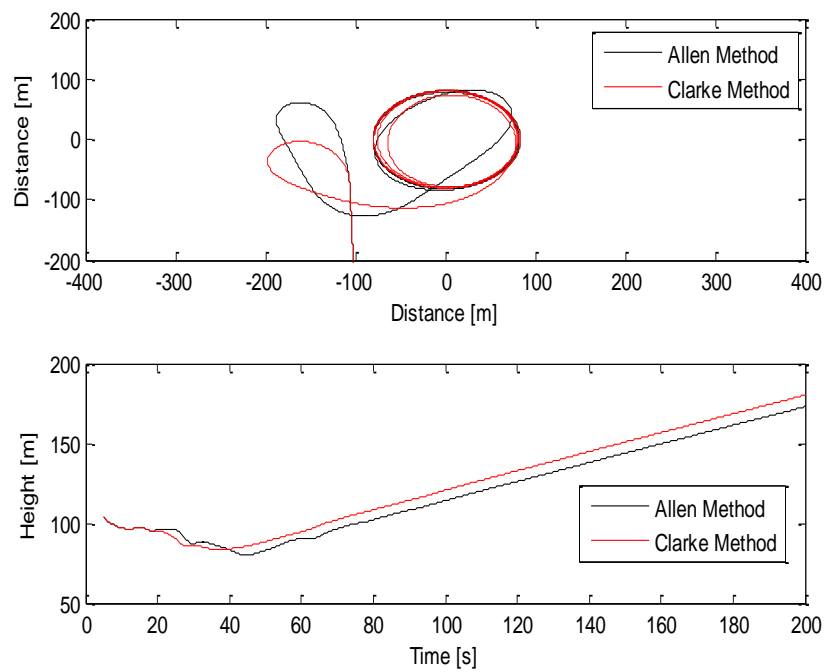

Figure 9. Perpormance Comparison

Although the Reichman-PD soaring controller shows improvements over Allen's method in the example quoted this is not always the case. This controller is of the same basic form as that used by Allen [2], with the exception that the autopilot he used could not accept bank angle commands, as a result his controller demands a turn rate. All that can be conclusively ascertained from the results is that the two methods are approximately equal. The advantage of the Reichman-PD soaring controller is that all of the terms in the controller have physical significance and are readily tuneable in real-time. Although the use of lowpass filters is more computationally intensive than other methods [1] [2] [3] it allows for dynamic adjustment of the number of datapoints used to predict the centre of the thermal. 


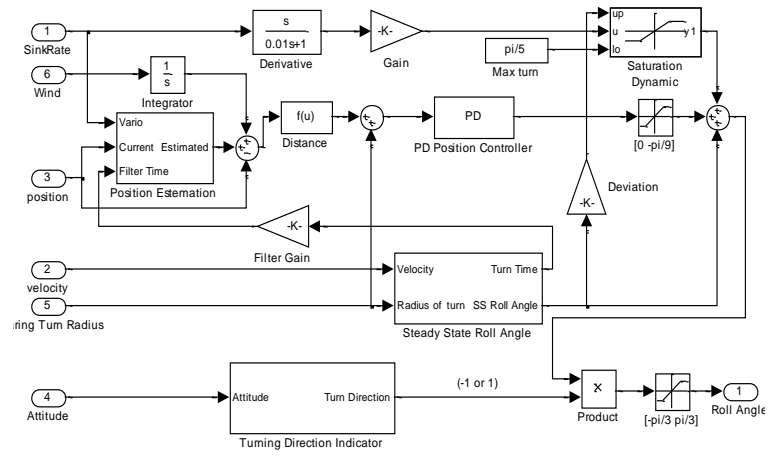

Figure 10. Soaring Control Structure

\section{Simulation RESUltS}

With the simulation environment in place it is possible to start investigating the feasibility of different mission profiles. The simulation scenario chosen for this exercise was a $20 \mathrm{~km}$ flight with a $10 \mathrm{knot}$ wind added $45^{\circ}$ to the desired track. A $45^{\circ}$ wind component is added because this is the worst case for the use of cloud streets. The atmospheric map used is time dependent, as detailed in section III. To put the results into context 4 approaches will now be compared; powered flight, soaring flight, soaring flight utilising cloud street phenomena and time constrained soaring flight.

The minimum save altitude was chosen as $500 \mathrm{ft}$. The cruse height was chosen as $1000 \mathrm{ft}$. This was also used as the power-on altitude for the soaring simulations as thermals bellow this height are more broken and harder to centre in, thus reflecting best practice for improving cross country performance. The MacCready function shown in Fig. 8 was used as this as felt to represent a typical UAV risk function. The MacCready function used is not ultimately risk averse as UAV generally do have motors and as such most applications have a speed-risk trade off. The impact of this function is to result in all the thermals encounter on some flights to be rejected in favour of cross country speed.

In order to get a reliable indication of the energy savings under these flight conditions and constraints, hundreds of flights were conducted with a spread of initial positions, although all were $20 \mathrm{~km}$ from the end location. Fig. 11 shows 3 typical soaring flights.
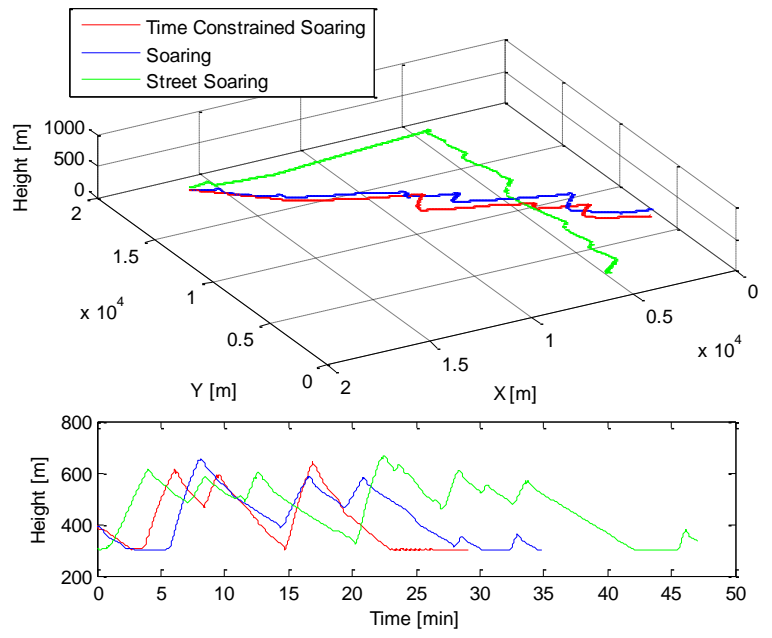

Figure 11. Simulated $20 \mathrm{~km}$ flight in $10 \mathrm{kt}$ wind

The relative performance of the 4 approaches can be summarised by discussing the following 3 plots; the energy consumption, distance travelled, and time taken on task.

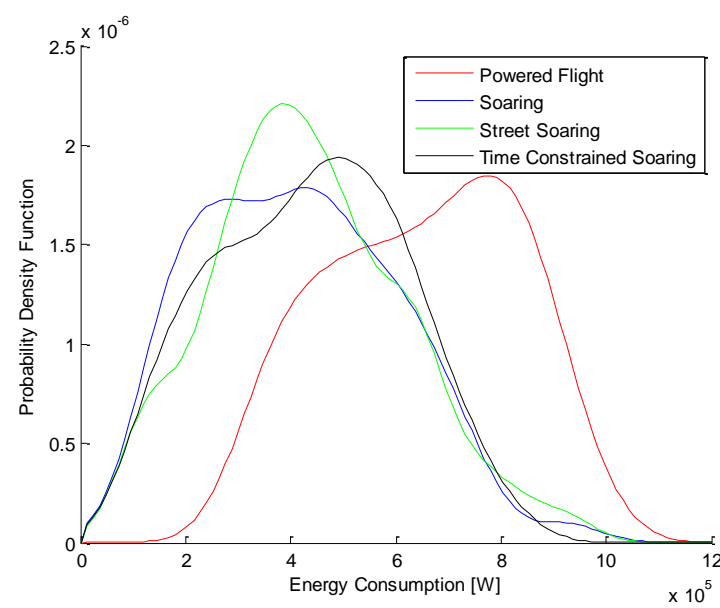

Figure $12 . \quad$ Energy Consumption

From Fig. 12 it is clear that there is a significant energy saving to be had by the use of atmospheric convection in the form of thermals. However there is a large variance in the amount of energy saved on each flight. The use of cloud streets provides the most reliable method for contacting thermals. It is worthy of note that the mean energy consumption of the street and non-street soaring methods are within $1 \%$ of each other. This is surprising as the wind vector is at the least favourable angle for street following, resulting is large increases in the distance travelled by the aircraft as can be seen from Fig. 13. This increase in distance flown causes the flight times to be substantially increased, as can be seen from Fig. 14.

The asymmetry in the power consumption in the powered flight is due to the fact that the aircraft was still allowed to extract energy from the thermals by slowing down in rising air, also known as 'Dolphining'. For the 
solely powered flights the aircraft was not allowed to turn in the rising air.

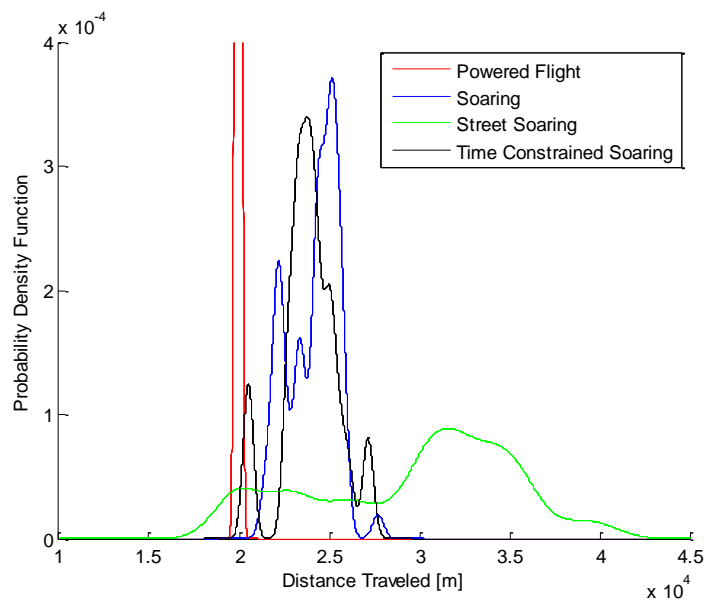

Figure 13. Distance Traveled

All of the soaring methods used inevitably increase the distance flown as the aircraft has to circle in rising pockets of air. This increase in distance travelled does not have to increase the time taken to complete the task. It is possible to use some, or all, of the energy gained to achieve a higher cross country speed.

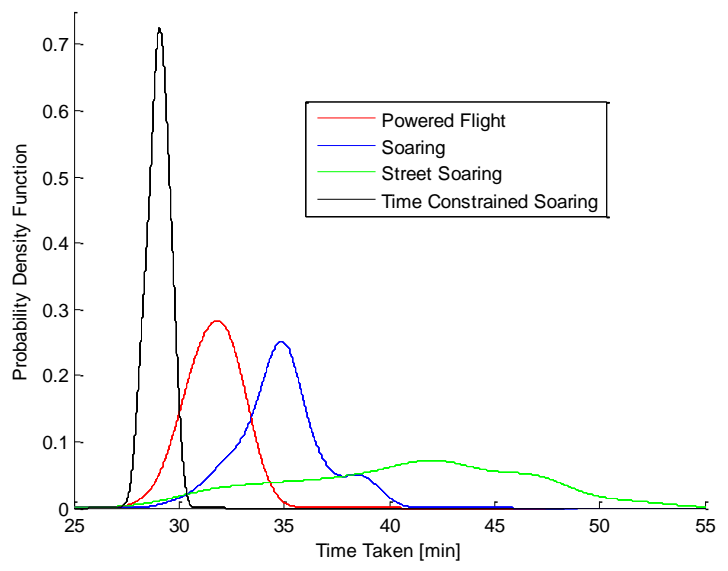

Figure 14. Time Taken on Task

If flight time is critical the MacCready function and therefore climb rate and cruse speed can be dynamically adjusted to constrain the maximum time on task. On the task presented the time constrained soaring approach guaranteed that the task would be completed in 30 minutes. Thus this time constrained approach not only completed the task on average $8 \%$ faster than the powered flight but used on average $33 \%$ less battery energy in the process.

\section{SUMMARY AND CONCLUDING REMARKS}

A highly detailed simulation model has been developed consisting of a non-linear aircraft dynamics model, a 4D parametric thermal model and a realistic control structure. The parametric thermal model was updated from those previously used to more accurately reflect the British climate. The presence of nontrivial amounts of sink associated with the thermal structure along with the prevalence of cloud streets has been reflected in the atmospheric model. The practical implementation of the MacCready function with restrictive height constraints has been discussed and implemented. A new flexible implementation of the Reichman centring technique was proposed and evaluated, providing promising results. These disparate elements were finally brought together in a simulated task. The simulated task was a $20 \mathrm{~km}$ outbound journey in challenging conditions. The MacCready risk function was setup to reflect the availability of the motor on the unmanned aerial vehicle. The use of cloud streets to help the probability of finding thermals was compared with purely opportunistic soaring. The use of thermal streets improved the chance of contacting a thermal and therefore resulted in a typical energy saving of 54\%, over purely power flight but increased the time taken to complete the task by up to $65 \%$, with a typical increase of $30 \%$. This variance in the time taken to complete the task is often undesirable. A purely opportunistic soaring approach resulted in a typical energy saving of $50 \%$ over purely power flight but increased the time taken to complete the task by up to $25 \%$, with a typical increase of $10 \%$. This variance in the time taken to complete the task is significantly smaller than that of a street following approach. If the time taken to complete the task is critical then streets can be used selectively in combination with the dynamic adjustment of the MacCready function to precisely set the cross country speed over large distances. This adjustment of cross country speed affords accurate control of the arrival time of the vehicle while maintaining large energy savings.

\section{REFERENCES}

[1] J. Wharington, and I. Herszberg, "Control of a High Endurance Unmanned Air Vehicle," ICAS-98-3,7,1, AIAA A98-31555, 21st ICAS Congress, Melbourne, Australia, September 13-18, 1998.

[2] M. J. Allen, "Autonomous Soaring for Improved Endurance of a Small Uninhabited Air Vehicle." NASA Dryden Flight Research Center, Edwards, California, 2005.

[3] D. J. Edwards, "Implementation Details and Flight Test Results of an Autonomous Soaring Controller". s.l. : North Carolina State University, Raleigh, NC, 27604, 2008.

[4] F. Irving, "The Paths of Soaring Flight". London : Imperial College press, 1999, 2006. ISBN 1-86094-055-2

[5] M. J. Allen, "Updraft Model for Development of Autonomous Soaring Uninhabited Air Vehicles" NASA Dryden Research Centre, Edwards, California, USA 2005

[6] J. A. Kyle, "Optimal Soaring by a Small Autonomous Glider". Oregon : Oregon State University, 2006.

[7] D. Piggott, "Gliding, A Handbook on Soaring Flight". London: A \& C Black, 2002, 1997, 1990, 1986, 1976, 1971, 1967, 1958. ISBN 0713661488

[8] C. E. Wallington, "Meteorology for Glider Pilots", Third International Edition, 1989 ISBN 0-7195-3303-1

[9] A. Welch, "Pilots' Whether". London: William Clowes and sons, Ltd, 1979, 1973. ISBN 0719526612.

[10] J. Delafield, "Gliding Competitively,1982, ISBN 0-71362224-5 
[11] D. H. Lenschow, and P. L. Stephens, "The Role of Thermals in the Convective Boundary Layer". s.l.: Boundary-Layer Meteorology, 1980

[12] P. B. MacCready, Jr, "Optimum airspeed selector", Soaring, pp. 10-11. 1958
[13] J. H. Cochrane, "MacCready Theory with Uncertain Lift and Limited Altitude". Technical Soaring, 23 (1999), pp. 88-96.

[14] I. D. Cowling, S. Willcox, Y. Patel, P. Smith. "Increasing persistence of UAVs and MAVs through thermal soaring". 1145, Clapham : The Aeronautical Journal, 2009, Vol 